\title{
The role of stress cardiac MRI imaging in patients with complete left bundle branch block
}

\author{
Balaji Rao', Yucheng Chen ${ }^{1,2^{*}}$, Raymond Kwong ${ }^{1}$ \\ From 15th Annual SCMR Scientific Sessions \\ Orlando, FL, USA. 2-5 February 2012
}

\begin{abstract}
Background
Left bundle branch block (LBBB) is a condition associated with increased mortality and poor prognosis, depending on the underlying etiology. Identification of the etiology and appropriate risk stratification of patients with LBBB is a challenge, despite the availability of various testing modalities. Nuclear exercise stress testing can show artificial perfusion defects in the interventricular septum. The artifacts are reduced but not completely excluded by use of vasodilator stress agents. In this study, we retrospectively reviewed the stress cardiac magnetic resonance (CMR) findings in patients with LBBB.
\end{abstract}

\section{Methods}

We retrospectively reviewed 49 consecutive patients with complete LBBB who underwent stress CMR. Of these 49 patients, 23 also underwent concurrent coronary angiography, 20 underwent nuclear stress test and 8 underwent all the three tests. The reference standard for flow limiting coronary artery disease (CAD) was coronary artery stenosis greater than $50 \%$ on coronary angiogram.

\section{Results}

Stress CMR correctly identified all patients $(n=13)$ with more than $50 \%$ coronary artery stenosis and all patients $(\mathrm{n}=7)$ without significant coronary artery stenosis.

Overall, the sensitivity, specificity, positive predictive value and negative predictive value of CMR in diagnosing flow limiting CAD were $100 \%, 70 \%, 81 \%$ and $100 \%$ respectively.

In patients who underwent both stress CMR and nuclear stress test $(n=20)$, stress CMR showed late gadolinium enhancement (LGE) in the coronary artery distribution in thirteen patients $(\mathrm{n}=13$ of 20$)$, consistent with fibrosis due to myocardial infarction. Where as nuclear stress test showed fixed defect in a coronary artery distribution in only six patients ( $\mathrm{n}=6$ of 20 ).

Stress CMR also demonstrated other etiologies like hypertrophic cardiomyopathy, cardiac iron overload and idiopathic dilated cardiomyopathy.

\section{Conclusions}

Stress CMR imaging has high sensitivity and negative predictive value in the identification of CAD, in patients with complete LBBB. It can also assess infiltrative cardiomyopathies. Stress CMR appears to provide a better comprehensive assessment than nuclear scintigraphy in patients with LBBB.

\section{Funding}

None.

\section{Author details}

${ }^{1}$ Non Invasive Cardiovascular Imaging, Brigham and Women's Hospital, Boston, MA, USA. ${ }^{2}$ Cardiology, West China Hospital, Chengdu, China.

Published: 1 February 2012

doi:10.1186/1532-429X-14-S1-P150

Cite this article as: Rao et al:: The role of stress cardiac MRI imaging in patients with complete left bundle branch block. Journal of Cardiovascular Magnetic Resonance 2012 14(Suppl 1):P150.

${ }^{1}$ Non Invasive Cardiovascular Imaging, Brigham and Women's Hospital,

Boston, MA, USA

Full list of author information is available at the end of the article

(c) 2012 Rao et al; licensee BioMed Central Ltd. This is an open access article distributed under the terms of the Creative Commons Attribution License (http://creativecommons.org/licenses/by/2.0), which permits unrestricted use, distribution, and reproduction in any medium, provided the original work is properly cited. 